\title{
The research output on interventions for the behavioural risk factors alcohol \& drug use and dietary risk is not related to their respective burden of ill health in countries at differing World Bank income levels
}

\author{
Carlo Frassetto ${ }^{*}$, Meisser \\ Madera $^{2 *}$, Maximilian Siebert ${ }^{3 *}$, \\ Karen Megranahan ${ }^{4 *}$, David \\ Roberts $^{5}$, Emma Plugge ${ }^{6}$ \\ ${ }^{1}$ School of Nursing, Catholic University of \\ Sacred Hearth, Rome, Italy \\ ${ }^{2}$ Department of Research, Faculty of \\ Dentistry, University of Cartagena, \\ Cartagena, Colombia \\ ${ }^{3}$ Univ Rennes, CHU Rennes, Inserm, CIC \\ 1414 (Centre d'Investigation Clinique de \\ Rennes), Rennes, France \\ ${ }^{4}$ Institute of Creative and Cultural \\ Entrepreneurship, Goldsmiths College, \\ University of London, London, UK \\ ${ }^{5}$ Oxford School of Public Health, \\ Nuffield Department of Population \\ Health, University of Oxford, Oxford, UK \\ ${ }^{6}$ Cochrane UK, Oxford, UK \\ * Joint first authorship.
}

\begin{abstract}
Background Alcohol and drug use (A\&D) and dietary risks are two increasingly important risk factors. This study examines whether there is a relationship between the burden of these risk factors in countries of specific income bands as defined by the World Bank, and the number of primary studies included in Cochrane Systematic Reviews (CSRs) conducted in those countries.
\end{abstract}

Methods Data was extracted from primary studies included in CSRs assessing two risk factors as outcomes. For each risk factor, data was obtained on its overall burden in disability-adjusted life years (DALYs) by World Bank Income Levels and examined for a link between DALYs, the number of primary studies and participants.

Results A total of 1601 studies from 95 CSRs were included. Only 18.3\% of the global burden for A\&D is in high income-countries (HICs) but they produced $90.5 \%$ of primary studies and include $99.5 \%$ of participants. Only $14.2 \%$ of the dietary risk burden is in HICs but they produced $80.5 \%$ of primary studies and included $98.1 \%$ of participants.

Conclusions This study demonstrates the unequal output of research heavily weighted towards HICs. More initiatives with informed contextual understanding are required to address this inequality and promote health research in low and middle-income countries.

\section{Correspondence to:}

Karen Megranahan MSc Institute for Creative and Cultural Entrepreneurship Goldsmiths College

University of London

New Cross

London SE14 6NW

UK

kmegr001@gold.ac.uk 
selected because Roberts et al showed that there was a higher than expected number of reviews given the disease burden addressing the risk factor of $A \& D$ but a lower than expected number addressing dietary risks. The Global Burden of Disease (GBD) study measures the burden of risk factors in disability-adjusted life years (DALYs), that is, the sum of years lived with disability and years of life lost to premature death. The 2016 GBD study shows behavioural risk factors accounted for $32.7 \%$ (95\% confidence interval 30.7-34.8) of attributable DALYs and highlights the importance of dietary risk factors and A\&D risk factors in LMICs as well as in high income countries (HICs) [4].

The aim is to determine whether the burden of each of these two risk factors in countries of a particular income band (defined by the World Bank), and the number of primary studies and of study participants included in Cochrane Systematic Reviews conducted in those countries are related.

\section{METHODS}

The Cochrane Database of Systematic Reviews via the Cochrane Library was searched using two filters (intervention and custom range date) to identify relevant systematic reviews published from $1^{\text {st }}$ January 2013 to the $2^{\text {nd }}$ October 2018. This five-year period was decided because it represents the median life expectancy of systematic reviews [5]. All primary intervention studies of any study design included in CSRs of interventions related to two modifiable behavioural risk factors (A\&D and dietary risks) were selected for data extraction. When several versions of a published review were identified, the most recent publication was considered. Protocols and systematic reviews about diagnosis, prognosis, methodology and overviews were excluded.

All titles and abstracts were independently screened to exclude irrelevant reviews. Full texts were obtained for a final decision where necessary. Any disagreements were resolved by discussion, and an additional reviewer participated until an agreement was reached. Each included CSR was independently reviewed by two of the team of reviewing authors (CF, KM, MS, MM) to identify primary studies assessing interventions addressing diet and A\&D.

The reviewing authors independently extracted data on the characteristics of primary studies including publication year, study design, country of first author, country where study took place, and number of participants. A 10\% random selection of the extracted data was checked by one of the other reviewers. For each risk factor assessed, we obtained data on its overall burden in DALYs by World Bank Country

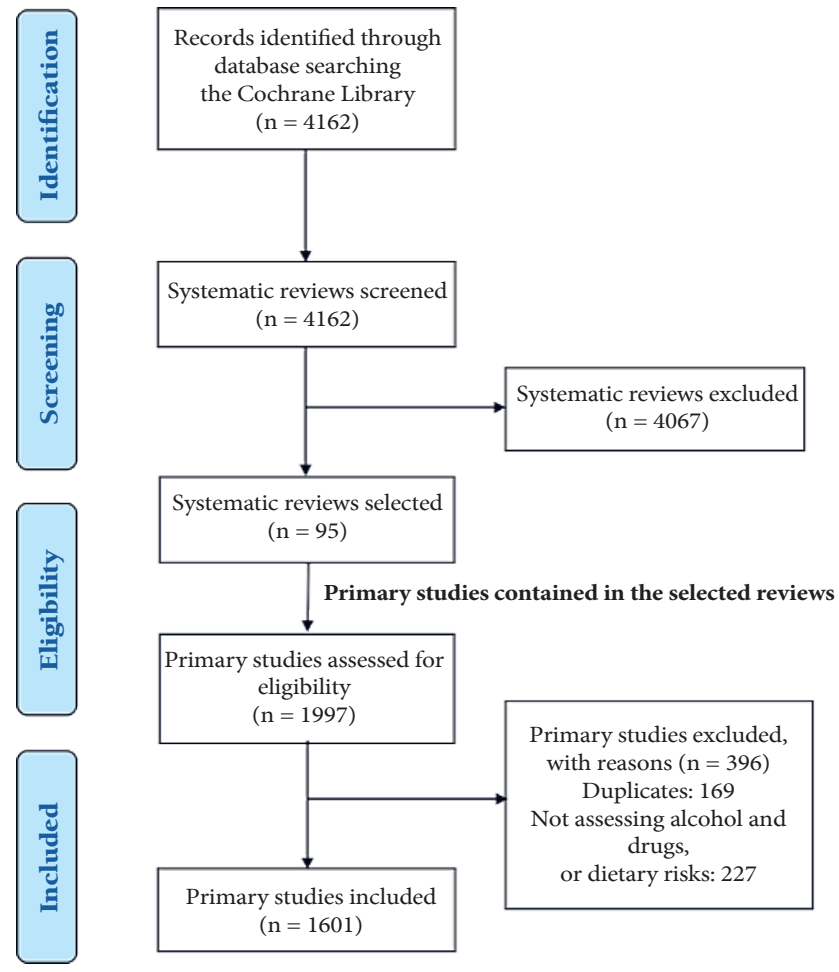

Figure 1. Prisma diagram of study selection.
Income Levels (WBIL) grouped into high, upper-middle, lower-middle and low income from the Global Burden of Disease study 2016 [6].

\section{Data analysis}

A descriptive analysis of characteristics of the included primary studies was performed. Data was analysed and presented in tables using frequency and percentage for categorical variables. We used the Chi squared Goodness of Fit (CSGOF) to test the null hypothesis that the number of primary studies and the number of DALYs for that risk factor grouped by income band (high income vs upper middle, lower middle and low-income countries) were of the same distribution. This was repeated for the number of study participants. All statistical analyses were performed with STATA version 11 software [7] assuming a significance level of 0.05 .

\section{RESULTS}

A total of 95 CSRs met the inclusion criteria; 45 (47.4\%) addressed dietary risks, 47 (49.5\%) addressed A\&D and three addressed (3.1\%) both factors; and included 1601 primary studies. The selection process of the systematic reviews and its respective primary studies is shown in Figure 1. 95.6\% of the primary studies were RCT's; whereas the A\&D sec- 
tion counted $97.1 \%$ RCTs and the dietary risks counted $94.3 \%$. A total of $86.8 \%$ of the included studies were conducted in HICs; in A\&D this made up a percentage $94.5 \%$ and in dietary risks $80.5 \%$. For further characteristics of primary studies included in Cochrane systematic reviews by assessed risk factors please refer to Table 1 .

The majority of the burden for A\&D was in LMICs but they only produced $5.5 \%$ of primary studies and $0.5 \%$ of participants were from these countries. Likewise, LMICs carried $85.8 \%$ of the burden for dietary risks but produced only $19.5 \%$ of primary studies and included $1.9 \%$ of participants. Details of the characteristics for the assessed risk factors by country income level can be found in Table 2.

The $\chi^{2}$ goodness of fit (CSGOF) test showed the number of primary studies was of a different distribution to both risk factors studied; A\&D risk factor burden in HICs and LMICs $(P<0.001)$, and dietary risk factor burden $(P<0.001)$. The number of study participants was also of a different distribution to the $A \& D$ risk factor burden in HICs and LMICs $(P<0.001)$ and also between dietary risk factor burden $(P<0.001)$.

\section{DISCUSSION}

The authors examined 1601 primary studies from 95 CSRs. The results show $85.8 \%$ of the burden of disease for dietary risks is borne by LMICs yet only 19.5\% of the primary studies and 1.9\% of participants

Table 1. Characteristics of primary studies included in Cochrane systematic reviews by assessed risk factors

\begin{tabular}{|c|c|c|c|}
\hline & ALCOHOL \& DRUG USE & Dietary risks & TOTAL \\
\hline Publication year & n (\%) & n (\%) & n (\%) \\
\hline $2011-2018$ & $194(26.7)$ & $369(42.1)$ & $563(35.2)$ \\
\hline $2001-2010$ & $356(49.1)$ & $320(36.5)$ & $676(42.2)$ \\
\hline $1991-2000$ & $131(18.1)$ & $142(16.2)$ & $273(17.0)$ \\
\hline$\leq 1990$ & $44(6.1)$ & $45(5.1)$ & $89(5.6)$ \\
\hline \multicolumn{4}{|l|}{ Study design: } \\
\hline Cross-sectional & $0(0)$ & $5(0.6)$ & $5(0.3)$ \\
\hline Cohort & $9(1.3)$ & $38(4.3)$ & $47(3.0)$ \\
\hline NRS & $1(0.1)$ & $6(0.7)$ & $7(0.4)$ \\
\hline Quasi-RCT & $11(1.5)$ & $1(0.1)$ & $12(0.7)$ \\
\hline RCT & $704(97.1)$ & $826(94.3)$ & $1530(95.6)$ \\
\hline \multicolumn{4}{|c|}{ Country (World Bank income level): } \\
\hline High & $685(94.5)$ & $705(80.5)$ & $1390(86.8)$ \\
\hline Upper middle & $33(4.5)$ & $89(10.2)$ & $122(7.6)$ \\
\hline Lower middle & $7(1.0)$ & $59(6.7)$ & $66(4.1)$ \\
\hline Low & $0(0)$ & $23(2.6)$ & $23(1.5)$ \\
\hline TOTAL & $725(100)$ & $876(100)$ & $1601(100)$ \\
\hline
\end{tabular}

NRS - no randomized controlled study, RCT - randomised controlled trial

Table 2. Characteristics for the assessed risk factors by country income level

\begin{tabular}{|c|c|c|c|c|}
\hline Country nncome level & DALYs, N IN MiLIons (\%) & Primary studies, n (\%) & $\begin{array}{l}\text { Country of PRIMARY STUdy } \\
1 \text { st AUthoR, n (\%) }\end{array}$ & $\begin{array}{l}\text { PabticipantS PRIMARY } \\
\text { StUdies, N (\%) }\end{array}$ \\
\hline \multicolumn{5}{|c|}{ Alcohol \& drugs use: } \\
\hline High & $24.8(18.3)$ & $685(94.5)$ & $689(95)$ & 469607 (99.5) \\
\hline Upper middle & $57.9(42.7)$ & $33(4.5)$ & $30(4.1)$ & $1929(0.4)$ \\
\hline Lower middle & $39.2(28.9)$ & $7(1.0)$ & $6(0.9)$ & $472(0.1)$ \\
\hline Low & $13.6(10)$ & $0(0)$ & $0(0)$ & $0(0)$ \\
\hline TOTAL & $135.5(100)$ & $725(100)$ & $725(100)$ & $472008(100)$ \\
\hline \multicolumn{5}{|l|}{ Dietary risks: } \\
\hline High & $32.4(14.2)$ & $705(80.5)$ & $738(84.2)$ & $530009(98.1)$ \\
\hline Upper middle & $91.8(40.2)$ & $89(10.2)$ & $87(10.0)$ & $6186(1.1)$ \\
\hline Lower middle & $92.2(40.3)$ & $59(6.7)$ & $44(5.0)$ & $3050(0.6)$ \\
\hline Low & $12.2(5.3)$ & $23(2.6)$ & $7(0.8)$ & $1256(0.2)$ \\
\hline TOTAL & $228.6(100)$ & $876(100)$ & $876(100)$ & $540501(100)$ \\
\hline
\end{tabular}

DALYs - disability-adjusted life years 
came from LMICs. The pattern is even starker for A\&D since $81.7 \%$ of the burden of disease related to $\mathrm{A} \& \mathrm{D}$ is borne by LMICs but only $5.5 \%$ of the primary studies and $0.5 \%$ of participants came from LMICs. Notably 1390 (86.8\%) of the 1601 primary studies examined were conducted in HICs. The total number of included studies taking place in the USA alone was 836, two thirds of those in HICs.

This study shows that the output of primary studies for the two behavioural risk factors, diet and A\&D, are not in line with their respective burden of ill health in countries at differing WBIL.

The findings were similar to the ones of other studies conducted around these two risk factors. A previous study had shown that there were a higher number of CSRs addressing A\&D than expected given risk factor disease burden [2], however, this study has identified that there is in fact a need for further research addressing $A \& D$ specifically in LMICs despite the fact that the risk factor of A\&D was 'over studied' at a global level.

Also shown is the globally 'understudied' risk factor of poor diet is severely 'understudied' in LMICs. The World Health Organisation's 2012 Strategy on Research for Health recognised the need to address "gaps in national and global research on health and health systems" [1]; this study has highlighted specific gaps and also the marked inequity in research outputs between HICs and LMICs.

Dietary risk is an increasing health issue for many countries [8] and is evident in countries of all income levels but for different reasons. In LMICs the reasons are particularly complex as populations in these countries experience under and over nutrition. Factors such as fast-food outlets, associated with poor diet and obesity in HICs, are increasingly an issue in LMICs, alongside poor sanitation or lack of food $[8,9]$. This highlights the urgent need for context specific research on effective interventions in these countries.

There is no doubt that $A \& D$ is on the increase $[10,11]$. Alcohol was reported as the $7^{\text {th }}$ highest risk factor for deaths in 2016 [12] and drug related overdose deaths in the USA alone had increased by 10\% per year since 1999 [13]. Again, this highlights the urgent need to develop the evidence base for effective interventions addressing A\&D in LMICs.

To the authors knowledge this is the first study that examines the burden of two behavioural risk factors, diet and $A \& D$, in countries of specific World Bank income bands and how it relates to the number of primary studies and their participants included in CSRs conducted in those countries. The study was rigorously conducted, with two reviewers looking at a sample of all data with $92.6 \%$ agreement.

Limitations of the study were that only primary studies eligible for inclusion in CSRs were considered and other systematic reviews could have been included. The sources of bias in the included systematic reviews was not investigated. Furthermore because of resource constraints, the reviewing authors were only able to examine two specific risk factors, dietary risk and $A \& D$, rather than all GBD level 2 modifiable risk factors. Finally, the identification of factors related to the burden of ill health is only exploratory. Several unmeasured confounders, for instance the difference in rural and urban areas, the cultural settings or the different quality of studies, could account for some of the findings. Other unmeasured confounders may exist, and great caution is warranted in interpreting these results, which naturally cannot be considered as reflecting any causal relationship.

The results of this research highlight that more primary research needs to be conducted in LMICs for the two behavioural risk factors studied; it should not be assumed that what is effective in HICs will also be effective in LMICs, although some interventions may indeed be globally relevant. This is part of a much wider problem regarding inequities in global health research. Factors hindering research equity in LMICs are worthy of further investigation such as sources of funding, language barriers to publication and broader socio-economic issues. HICs can support justice in global health by the design of grant programmes that will help adjust the disparity [14]. Clearly initiatives such as increased collaboration, capacity building initiatives for health research in LMICs, increased access to related literature and more funding opportunities for LMICs will help to start to address this imbalance [15].

In conclusion, this study demonstrates the inequal output of research heavily weighted towards HICs for $A \& D$ and Dietary risks. Therefore, more initiatives with informed contextual understanding are required to address this inequality and promote health research in low and middle-income countries. 


Acknowledgements: The authors thank Anne Eisinga for her contribution to this work. This work was conducted
during a student elective period at Cochrane UK by the four first authors. Cochrane UK provided office space during
the elective period for the reviewing authors.
Disclaimer: The views expressed in the submitted article are those of the authors and not an official position of the
institutions to which the authors belong.
Funding: No funding was received.
Authorship contributions: EP and DR conceived the initial design, which was subsequently refined by CF, MM, KM
and MS. CF, MM, KM and MS gathered the data which was analysed by MM, MS, EP and DR. The interpretation was
agreed by all authors. CF, MM, KM and MS drafted the manuscript which was critically revised by all authors and agree
now to be accountable for all aspects of the work in ensuring that questions related to the accuracy or integrity of any
part of the work are appropriately investigated and resolved.
Competing interests: EP is employed on a consultancy basis by Cochrane UK. The authors completed the ICMJE
Unified Competing Interest form (available upon request from the corresponding author), and declare no further con-
flicts of interest.

1 World Health Organisation. The WHO strategy on research for health. 2012. Available: https://www.who.int/phi/WHO_ Strategy_on_research_for_health.pdf. Accessed: 25 November 2018.

2 Roberts DJ, Cafferkey J, Plugge E. Cochrane systematic reviews of interventions for risk factors correlate weakly with global risk factor burden: a cross-sectional study. J Clin Epidemiol. 2018;97:103-10. Medline:29288132 doi:10.1016/j. jclinepi.2017.12.002

3 Data.worldbank.org. World Bank Open Data | Data. 2018. Available: https://data.worldbank.org/. Accessed: 25 November 2018.

4 GBD 2016 Risk Factors Collaborators. Global, regional, and national comparative risk assessment of 84 behavioural, environmental and occupational, and metabolic risks or clusters of risks, 1990-2016: a systematic analysis for the Global Burden of Disease Study 2016. Lancet. 2017;390:1345-422. Medline:28919119 doi:10.1016/S0140-6736(17)32366-8

5 Shojania KG, Sampson M, Ansari MT, Ji J, Doucette S, Moher D. How quickly do systematic reviews go out of date? A survival analysis. Ann Intern Med. 2007;147:224-33. Medline:17638714 doi:10.7326/0003-4819-147-4-20070821000179

6 Global Health Data Exchange. Global Burden of Disease Study. 2016. Available: http://ghdx.healthdata.org/gbd-2016. Accessed: 23 October 2018.

7 STATA Corp. STATA® version 11. STATA Corp.

8 World Health Organization, editor. Obesity: preventing and managing the global epidemic: report of a WHO consultation. Geneva: World Health Organization; 2000.

9 Popkin BM. Nutritional Patterns and Transitions. Popul Dev Rev. 1993;19:138. doi:10.2307/2938388

10 Stevens G, Mascarenhas M, Mathers C. Global health risks: progress and challenges. Bull World Health Organ. 2009;87:646. Medline:19784438 doi:10.2471/BLT.09.070565

11 Gowing LR, Ali RL, Allsop S, Marsden J, Turf EE, West R, et al. Global statistics on addictive behaviours: 2014 status report: Addiction global statistics. Addiction. 2015;110:904-19. Medline:25963869 doi:10.1111/add.12899

12 GBD 2016 Alcohol Collaborators. Alcohol use and burden for 195 countries and territories, 1990-2016: a systematic analysis for the Global Burden of Disease Study 2016. Lancet. 2018;392:1015-35. Medline:30146330 doi:10.1016/ S0140-6736(18)31310-2

13 Hedegaard H, Warner M, Minino AM. Drug overdose deaths in the United States, 1999-2015. NCHS Data Brief. 2017;(237):1-8. Medline:28256996

14 Pratt B, Hyder AA. Ethical responsibilities of health research funders to advance global health justice. Glob Public Health. 2019;14:80-90. Medline:29724152 doi:10.1080/17441692.2018.1471148

15 Langer A, Díaz-Olavarrieta C, Berdichevsky K, Villar J. Why is research from developing countries underrepresented in international health literature, and what can be done about it? Bull World Health Organ. 2004;82:802-3. Medline:15643806 